\title{
Premature Atrial Complex With P'R Prolongation Indicates Multiple Atrioventricular Nodal Pathways
}

\author{
Etsuko Fukuda, BS; Takeshi Yamashita, MD; Kumiko Tamakoshi; Kouichi Sagara, MD; \\ Hiroyuki Iinuma, MD; Tadanori Aizawa, MD; Long-Tai Fu, MD
}

\begin{abstract}
Background The goal of the present study was to test if ambulatory Holter recordings can predict the electrophysiologic study (EPS) findings in patients with supraventricular tachycardia (SVT).

Methods and Results The study involved 110 patients with SVT who underwent Holter recording, and then EPS. The hypotheses were that (1) a P'R interval of premature atrial complexes (PACs) between 280 and $400 \mathrm{~ms}$ in the Holter recordings predicted dual atrioventricular nodal (AVN) pathways, (2) P'R interval $>400 \mathrm{~ms}$ predicted triple or more AVN pathways, and (3) SVT initiated by a single PAC suggested easy SVT induction during the EPS. The EPS revealed dual AVN pathways in 14 (93\%) of 15 patients with P'R intervals between 280 and $400 \mathrm{~ms}$ on the Holter recordings, and triple or more AVN pathways in $18(90 \%)$ of 20 patients with P'R intervals $>400 \mathrm{~ms}$. In addition, a single extrastimulus easily induced SVT during the EPS in $11(85 \%)$ of 13 patients in whom SVT was initiated by a single PAC during Holter recording.

Conclusion The ambulatory Holter recording criteria specifically predicted the EPS findings, thereby provid-
\end{abstract} ing useful advance information. (Circ J 2005; 69: 1233-1236)

Key Words: Ambulatory Holter recording; Electrophysiologic study; Multiple atrioventricular nodal pathways; Supraventricular tachycardia

$\mathbf{M}$ any previous studies have shown not only 2 but also 3 or more atrioventricular nodal (AVN) pathways as part of the reentrant circuit of AVN reentrant tachycardia (AVNRT)!-6 Moreover, AV reciprocating tachycardia (AVRT) involving these multiple AVN pathways as the descending limb of the reentrant circuit has been observed? Although these multiple AVN pathways have been diagnosed only by electrophysiologic study (EPS), ambulatory Holter recording, which is usually performed before invasive testing, may provide some relevant information. To examine whether this is so, we studied the relationship between preceding ambulatory Holter recordings and the EPS findings in patients with supraventricular tachycardia (SVT).

\section{Methods}

\section{Study Population}

From December 1993 to September 2002, 110 patients (54 men, 56 women, aged 55 \pm 16 years) with SVT (56 patients with AVNRT, 54 patients with AVRT) underwent an EPS with preceding ambulatory Holter recording. Thirtyfive of these patients had manifest Wolff-Parkinson-White (WPW) syndrome during normal sinus rhythm. All patients gave informed consent before the EPS.

\section{Definition of Multiple AVN Pathways \\ In our previous EPS of multiple AVN pathways, we showed that the atrio-His $(\mathrm{AH})$ intervals differ between the}

(Received March 14, 2005; revised manuscript received June 27, 2005; accepted July 7, 2005)

The Cardiovascular Institute, Tokyo, Japan

Mailing address: Etsuko Fukuda, BS, The Cardiovascular Institute, Roppongi 7-3-10, Minato-ku, Tokyo 106-0032, Japan. E-mail: etsukof@cvi.or.jp fast, intermediate, and slow pathways and that the conduction time through each pathway varies within almost constant ranges independent of the basic cycle length? In that study, the AH conduction times through the fast, intermediate, and slow AVN pathways were $<240 \mathrm{~ms}$, between $240 \mathrm{~ms}$ and $360 \mathrm{~ms}$, and $>360 \mathrm{~ms}$ at cycle lengths of both 600 and $400 \mathrm{~ms}$. We applied those results to the present ambulatory Holter recordings with a roughly estimated P-atrial electrogram interval of $40 \mathrm{~ms}$. Using the longest P'R interval of the premature atrial complex (PAC) in each patient, we defined the fast, intermediate, and slow AVN pathways in the ambulatory Holter recordings as follows: (1) P'R interval between $280 \mathrm{~ms}$ and $400 \mathrm{~ms}$ represents the existence of dual AVN pathways (fast and intermediate pathways), and (2) P'R interval $>400 \mathrm{~ms}$ represents the existence of triple or more AVN pathways (fast, intermediate, and slow pathways). All P'R intervals with narrow QRS complexes during the ambulatory Holter recordings were measured manually at a paper speed of $25 \mathrm{~mm} / \mathrm{s}$, and the longest P'R interval was determined in each patient.

A conventional EPS was performed when multiple AVN pathways were confirmed by the following criteria: (1) the presence of discrete discontinuities in the AVN conduction curve with sudden jumps of at least $50 \mathrm{~ms}$ with a $10-\mathrm{ms}$ decrease in the coupling interval using up to triple atrial extrastimuli, and/or (2) the presence of spontaneous transformation between the intermediate-fast, slow-fast, and slow-intermediate forms of AVNRT? In patients with AVRT, the electrophysiologic characteristics of the AV node were determined after catheter ablation of the accessory pathways. We also determined the induction pattern of SVT and analyzed the relationship between the initiation pattern observed in the ambulatory Holter recordings and that observed in the EPS. 
Table 1 Ambulatory Holter Recording and Electrophysiologic Study Findings

\begin{tabular}{lccc}
\hline \hline & $\begin{array}{c}A V N R T \\
(n=56)\end{array}$ & $\begin{array}{c}\text { AVRT } \\
(n=54)\end{array}$ & $\begin{array}{c}\text { Total } \\
(n=110)\end{array}$ \\
\hline Ambulatory Holter recording & & & \\
P'R interval between $280 \mathrm{~ms}$ and $400 \mathrm{~ms}$ & 12 & 3 & 15 \\
P'R interval more than $400 \mathrm{~ms}$ & 20 & 0 & 13 \\
SVT initiated by a single PAC & 8 & 5 & 36 \\
Electrophysiologic study & 21 & 15 & 35 \\
Dual AVN pathways & 35 & 0 & 62 \\
Triple or more AVN pathways & 28 & 34 & \\
SVT induced by single atrial extrastimulus & & & \\
\end{tabular}

AVNRT, atrioventricular nodal reentrant tachycardia; AVRT, atrioventricular reciprocating tachycardia; SVT, supraventricular tachycardia; PAC, premature atrial complex; AVN, atrioventricular node.

\section{Statistical Analysis}

Sensitivity, specificity, and positive and negative predictive values were calculated by the conventional method ${ }^{9}$ for the Holter recordings criteria in relation to the EPS findings.

\section{Results}

\section{Ambulatory Holter Recordings (Table 1)}

In the preceding ambulatory Holter recordings, the patients had 741 33,921 PACs/day (from 1 to 39,422 PACs/day). Whereas SVT of more than 3 beats was observed in 66 patients $(60 \%)$ in the ambulatory Holter recordings, clinical spontaneous SVT lasting 30s or more was observed in 23 patients (20.9\%). All P'R intervals were less than $600 \mathrm{~ms}$. The longest P'R interval was between $280 \mathrm{~ms}$ and $400 \mathrm{~ms}$ (Fig 1A) in 15 patients $(13.6 \%$ ) and $>400 \mathrm{~ms}$ (Fig 1B) in 20 patients $(18.2 \%)$. SVT was initiated by a single PAC (Fig 1C,D) in 13 patients (11.8\%).

\section{EPS (Table 1)}

Dual but not triple AVN pathways were confirmed in 36 patients $(32.7 \%, 21$ with AVNRT and 15 with AVRT). The triple or more AVN pathways were observed in 35 patients (31.8\%), all of whom had AVNRT. SVT was induced by a single atrial extrastimulus in 62 patients (56.3\%).

\section{Relationship Between the Ambulatory Holter Recordings and the EPS Findings}

The appearance of a P'R interval between $280 \mathrm{~ms}$ and $400 \mathrm{~ms}$ during the ambulatory Holter recordings was strongly associated with existence of dual AVN pathways: the EPS conformed dual AVN pathways in 14 of 15 patients $(93.3 \%)$ with these PACs, whereas dual AVN pathways existed in 22 of 95 patients (23.2\%) without a P'R interval between $280 \mathrm{~ms}$ and $400 \mathrm{~ms}$. A P'R interval $>400 \mathrm{~ms}$ well predicted the existence of triple or more AVN pathways: the EPS revealed triple or more AVN pathways in 18 of 20 patients $(90 \%)$ with these PACs, compared with 17 of 90 patients $(18.9 \%)$ without a P'R interval $>400 \mathrm{~ms}$. Moreover, the SVT initiation pattern during the ambulatory Holter recordings associated well with the EPS findings: of the 13 patients in whom SVT was spontaneously initiated by a single PAC during ambulatory Holter recording, 11 $(84.6 \%)$ showed SVT induced by a single atrial extrastimulus during the EPS.

The specificity and sensitivity of the ambulatory Holter recording criteria are shown in Table 2 . When limited to patients without manifest WPW syndrome, the specificities of these criteria regarding P'R interval were not affected:
98\% for dual and $97 \%$ for triple or more AVN pathways. Although their sensitivities were relatively low, our 3 parameters were highly specific, predictive of the results of the EPS, irrespective of the existence of manifest WPW syndrome.

\section{Discussion}

Although ambulatory Holter recordings are useful for identifying various types of arrhythmias ${ }^{10}$ evaluation of the AVN characteristics is usually done by an invasive EPS. However, based on our evaluation of the relationship between the ambulatory Holter recordings and the EPS findings, we have shown that ambulatory Holter recording is also useful for investigating the AVN. The appearance of prolonged P'R intervals in the ambulatory Holter recordings well predicts the characteristics of AVN conduction manifested in the EPS.

Multiple AVN pathways are proven by the presence of 2 or 3 discrete discontinuities in the AVN conduction curve generated during an EPS and are known to exist in 5-10\% of patients with SVT ${ }^{-3}$ In the present study, we observed triple or more AVN pathways in $31.8 \%$ of patients, a slightly higher percentage than expected. However, because we constructed AV conduction curves using up to 3 atrial extrastimuli, the results are thought to be quite accurate. In addition, spontaneous transformation between the intermediate-fast, slow-fast, and slow-intermediate forms of AVNRT was included.

In the EPS, atrial extrastimuli are introduced with varying coupling intervals at fixed basic cycle lengths, whereas in ambulatory Holter recording, the PAC occurs with varying coupling intervals at various cycle lengths of sinus rhythm. It might seem as though the conditions cannot be compared, but the P'R interval is known to be affected mainly by the basic cycle length and also by the coupling interval of the PAC. However, in the present analysis, we emphasized the zone of the longest P'R interval. Our previous study confirmed that the conduction times through the AVN pathways are dependent on the basic cycle length and the coupling intervals of the extrastimuli, but that the $\mathrm{AH}$ intervals through each pathway can vary within almost constant ranges independent of the basic cycle length and the coupling interval. Therefore, we hypothesized that the longest P'R interval would predict the existence of multiple AVN pathways and our study revealed that the ambulatory Holter recordings could predict the AVN characteristics observed in the subsequent EPS with high specificity.

In addition to the characteristics of AVN conduction, we 


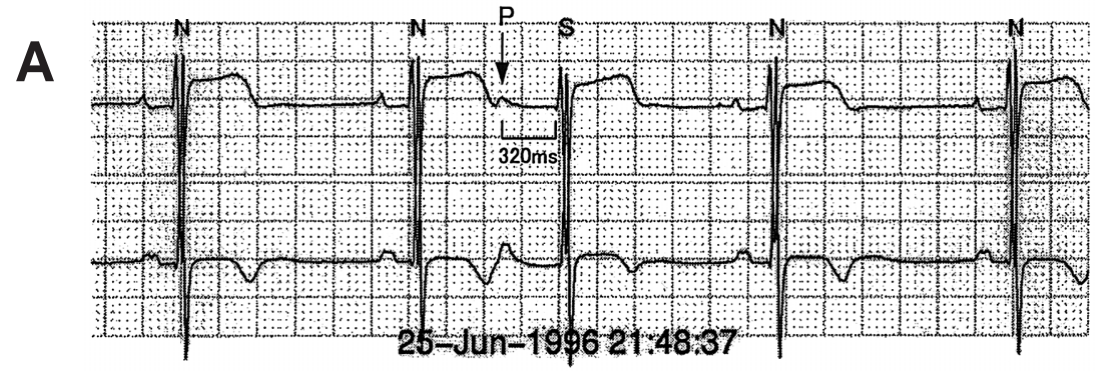

B

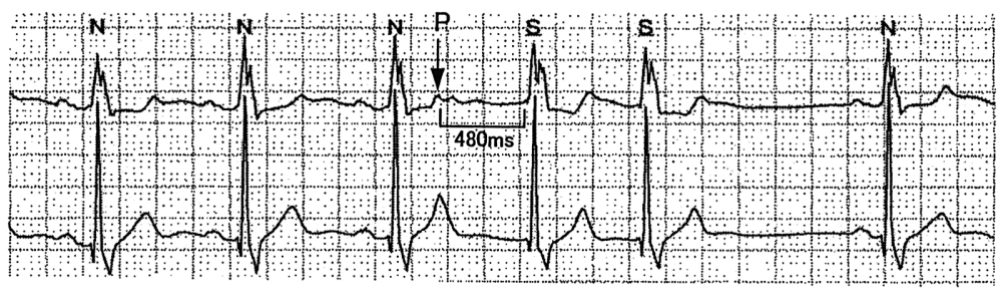

C

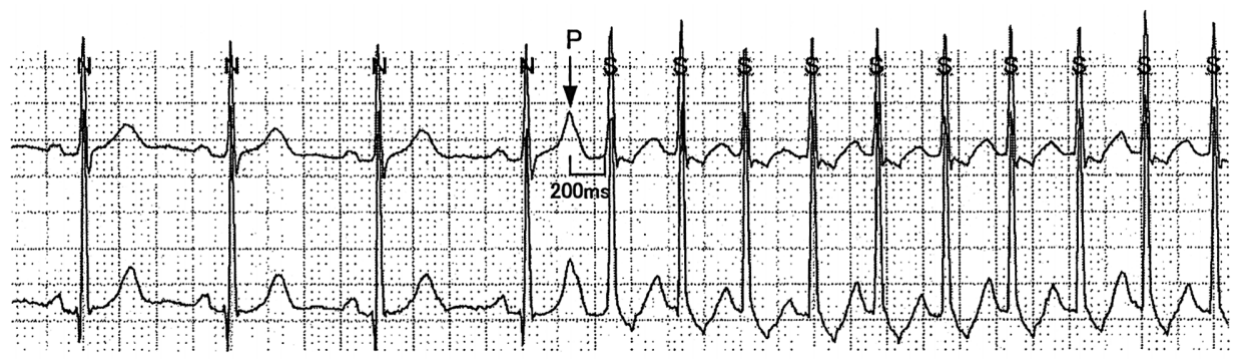

D

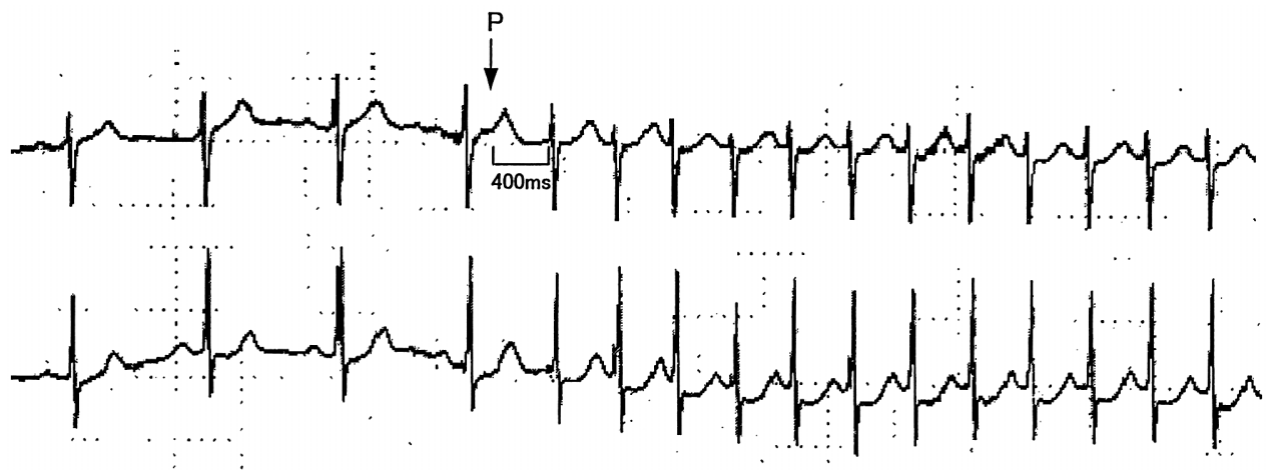

Fig 1. (A) Representative ambulatory Holter recording of a P'R interval of premature atrial complex (PAC) between $280 \mathrm{~ms}$ and $400 \mathrm{~ms}$. In this case, the P'R interval of the PAC was $320 \mathrm{~ms}$, predictive of dual atrioventricular nodal (AVN) pathways. (B) Representative ambulatory Holter recording of a P'R interval of PAC of more than $400 \mathrm{~ms}$. In this case, the P'R interval of PAC was $480 \mathrm{~ms}$, predictive of triple or more AVN pathways. (C) Representative ambulatory Holter recording of initiation of atrioventricular reciprocating tachycardia by a single PAC with a P'R interval of $200 \mathrm{~ms}$. Retrograde $\mathrm{P}$ waves were observed in the ST segments, suggesting the existence of concealed accessory pathways. (D) Representative ambulatory Holter recording of initiation of AVN reentrant tachycardia by a single PAC with a P'R interval of $400 \mathrm{~ms}$, suggesting the existence of triple AVN pathways.

Table 2 Relationship Between Ambulatory Holter Recording and Electrophysiologic Study Findings

\begin{tabular}{|c|c|c|c|c|c|}
\hline Ambulatory Holter recording & Electrophysiologic study & $\begin{array}{l}\text { Specificity } \\
(\%)\end{array}$ & $\begin{array}{l}\text { Sensitivity } \\
(\%)\end{array}$ & $\begin{array}{l}P P V \\
(\%)\end{array}$ & $\begin{array}{l}N P V \\
(\%)\end{array}$ \\
\hline$P^{\prime} R$ interval between $280 \mathrm{~ms}$ and $400 \mathrm{~ms}$ & Dual AVN pathways & 98 & 39 & 93 & 76 \\
\hline$P^{\prime} R$ interval $>400 \mathrm{~ms}$ & $\begin{array}{l}\geq \text { triple or more AVN } \\
\text { pathways }\end{array}$ & 97 & 51 & 90 & 81 \\
\hline SVT initiated by a single PAC & $\begin{array}{l}\text { SVT induced by a single } \\
\text { atrial extrastimulus }\end{array}$ & 96 & 18 & 85 & 47 \\
\hline
\end{tabular}

PPV, positive predictive value; NPV, negative predictive value. All other abbreviations, see Table 1. 
were able to predict the induction pattern of SVT in the EPS on the basis of the ambulatory Holter recordings. In most patients with SVT initiated by a single PAC during ambulatory Holter recording, a single atrial extrastimulus also induced tachycardia in the EPS, as expected. However, we do not fully understand the relationship between the 2 events. Feasibility of SVT induction in the EPS depends on several factors: the refractory periods of the antegrade and retrograde limbs and the conduction time through the AVN, all of which would be affected by the basic cycle length, the coupling intervals of the extrastimuli, and the autonomic nervous system. Because the ambulatory Holter recordings do not well reflect these important factors individually, the observed relationship should be indirect. In patients with inducible SVT, SVT might occur frequently during daily life, thus increasing the chances of catching an episode during ambulatory Holter recording. Therefore, it should be noted that SVT initiated by a single PAC in the ambulatory Holter recordings may not be a good marker for predicting the feasibility of SVT induction compared with predicting the presence of multiple AVN pathways based on the P'R interval.

\section{Study Limitations}

First, because the ambulatory Holter recordings are electrocardiograms recorded during daily life, noise can not be avoided completely. Moreover, it would be difficult to determine the P'R intervals of occasional low amplitude $\mathrm{P}$ waves. We had to omit the recordings with much noise and/or extremely low amplitude $\mathrm{P}$ waves and thus the sensitivity of the specific findings was decreased. Second, in 35 patients with manifest WPW syndrome, P'R intervals with pre-excitation were excluded. Our criteria for the P'R intervals can be applied only to narrow QRS complexes. Third, the sensitivity of our findings was quite low; however, this limitation is intrinsic to ambulatory Holter recording itself!1 ${ }^{1}$ Similarly, ambulatory Holter recordings without PAC and SVT could never be useful, a limitation of non-invasive testing. Nevertheless, we believe ambulatory Holter recordings can aid in the assessment of AVN characteristics in SVT patients.

\section{References}

1. Lee KL, Chun HM, Liem LB, Lauer MR, Young C, Sung RJ. Multiple atrioventricular nodal pathways in humans: Electrophysiologic demonstration and characterization. J Cardiovasc Electrophysiol 1998; 9: 129-140.

2. Tai CT, Chen SA, Chiang CE, Lee SH, Chiou CW, Ueng KC, et al. Multiple anterograde atrioventricular node pathways in patients with atrioventricular node reentrant tachycardia. J Am Coll Cardiol 1996; 28: $725-731$.

3. Swiryn S, Bauernfeind RA, Palileo EA, Strasberg B, Duffy CE, Rosen KM. Electrophysiologic study demonstrating triple antegrade AVN pathways in patients with spontaneous and/or induced supraventricular tachycardia. Am Heart J 1982; 103: 168-176.

4. Dopirak MR, Schaal SF, Leier CV. Triple AVN pathways in man? J Electrocardiol 1980; 13: 185-188.

5. Manita M, Kaneko Y, Kurabayashi M, Yeh SJ, Wen MS, Wang CC, et al. Electrophysiological characteristics and radiofrequency ablation of accessory pathways with slow conductive properties. Circ $J$ 2004; 68: 1152-1159.

6. Hara H, Niwano S, Yoshida T, Matsuyama N, Wakisaka Y, Sasaki T, et al. Prediction of clinical recurrence of atrioventricular-nodal reentrant tachycardia (AVNRT) after successful slow pathway ablation. Circ J 2004; 68: 558-562.

7. Shinohara A, Bando S, Ikefuji H, Yamamoto K, Nishikado A, Ito S. A case of concealed WPW syndrome with three different reciprocal tachycardia due to triple AVN pathways. Tokushima J Exp Med 1992; 39: 149-155.

8. Yamashita T, Murakawa Y, Ajiki K, Omata M. Triple atrioventricular nodal pathways can be masked. Am J Cardiol 1996; 77: $1365-$ 1367.

9. Armitage P, Berry G. Statistical methods in epidemiology. In: Statistical methods in medical research, $3^{\text {rd }}$ edn. Cambridge: Blackwell Science; 1994; 507-534.

10. Wellens HJ, Gorgels AP, Smeets JL, den Dulk K. Ambulatory electrocardiography evaluation of supraventricular tachyarrhythmia and bradyarrhythmia. Cardiol Clin 1992; 10: 361 - 370.

11. Shenasa M, Nadeau R, Savard P, Lemieux R, Curtiss EI, Follansbee WP. Noninvasive evaluation of supraventricular tachycardias. Cardiol Clin 1990; 8: 443-464. 